\title{
Solving vehicle routing problems with asymmetric costs and heterogeneous fleets
}

\author{
Rosa Herrero* \\ Telecommunications and Systems Engineering Department, \\ Autonomous University of Barcelona, \\ Edifici Q, 08193 Bellaterra, Cerdanyola del Vallès, Spain \\ E-mail: rherrero.math@gmail.com \\ *Corresponding author
}

\section{Alejandro Rodríguez}

Departamento de Organización de Empresas,

Universitat Politècnica de València,

Pza. Ferrándiz i Carbonell 2, 03801 Alcoi, Spain

E-mail: arodriguez@doe.upv.es

\section{José Cáceres-Cruz and Angel A. Juan}

Computer Science Department,

IN3-Open University of Catalonia,

Roc Boronat, 117 - 08018 Barcelona, Spain

E-mail: jcaceresc@uoc.edu

E-mail: ajuanp@uoc.edu

\begin{abstract}
The vehicle routing problem (VRP) is a flourishing research area with clear applications to real-life distribution companies. However, most VRP-related academic articles assume the existence of a homogeneous fleet of vehicles and/or a symmetric cost matrix. These assumptions are not always reasonable in real-life scenarios. To contribute in closing this gap between theory and practice, we propose a hybrid methodology for solving the asymmetric and heterogeneous vehicle routing problem (AHVRP). In our approach, we consider: 1) different types of vehicle loading capacities (heterogeneous fleets); 2) asymmetric distance-based costs. The proposed approach combines a randomised version of a well-known savings heuristic with several local searches specifically adapted to deal with the asymmetric nature of costs. A computational experiment allows us to discuss the efficiency of our approach and also to analyse how routing costs vary when slight departures from the homogeneous fleet assumption are considered.
\end{abstract}

Keywords: real-life vehicle routing problem; heterogeneous fleets; asymmetric costs; randomised algorithms.

Reference to this paper should be made as follows: Herrero, R., Rodríguez, A., Cáceres-Cruz, J. and Juan, A.A. (2014) 'Solving vehicle routing problems with asymmetric costs and heterogeneous fleets', Int. J. Advanced Operations Management, Vol. 6, No. 1, pp.58-80. 
Biographical notes: Rosa Herrero is a $\mathrm{PhD}$ student of the Industrial Engineering and Advanced Production Techniques at the Autonomous University of Barcelona. She has a Mathematics degree at the Autonomous University of Barcelona. She also completed predoctoral internships at the University of Ferrara collaborating on a project to solve a real-life health home care problem. Her main research interests are related to optimisation of vehicle routing on realistic scenarios, and her research interests include hybridisation of different techniques such as lagrangian relaxation, constraint programming and metaheuristics.

Alejandro Rodríguez received his $\mathrm{PhD}$ in Management Engineering from Polytechnic University of Valencia. He is a Professor at the Department of Management, Polytechnic University of Valencia. His research interests include issues related to operations research and the management sciences, and vehicle routing problems. He has published research papers at the national and international journals, and conference proceedings, two of which have been published in Computers and Operations Researches.

José Cáceres-Cruz is a $\mathrm{PhD}$ student of the Information and Knowledge Society Doctoral Programme at the Open University of Catalonia. He received his Specialisation on Information Systems from the Central University of Venezuela and his Computer Engineering degree from the Simón Bolívar University. His main research interests are related to optimisation of vehicle routing on realistic scenarios and simulation solutions.

Angel A. Juan is an Associate Professor of Applied Optimisation, Simulation and Data Analysis in the Computer Science Department at the Open University of Catalonia (UOC). He is also a Researcher at the Internet Interdisciplinary Institute (IN3). He holds a $\mathrm{PhD}$ in Applied Computational Mathematics (UNED) and an MS in Applied Mathematics (University of Valencia). His research interests include applied optimisation, randomised heuristics and computer simulation. He has published over 100 peer-reviewed papers regarding these fields. Currently, he is the coordinator of the CYTEDHAROSA Network and editorial board member of several international journals. His webpage is: http://ajuanp.wordpress.com.

\section{Introduction}

Vehicle routing problems (VRPs) deal with the physical distribution of goods from a central depot to customers, see for instance, Toth and Vigo (2002) and Golden et al. (2008). The best-known VRP variant is the so-called capacitated vehicle routing problem (CVRP). In the CVRP it is assumed the existence of a homogeneous fleet of vehicles with limited capacity. Another frequent assumption is that distance-based costs associated with travelling from one node $i$ (customer or depot) to another node $j, c_{i j}$, are symmetric, i.e., $c_{i j}=c_{j i}$ for all pair of nodes. A wide number of VRP variants have been developed during the last years, each of them considering different sets of characteristics and constraints. Usually, the main goal of the CVRP is to minimise distance-based costs associated with the distribution of products among customers while satisfying customers" demands. 
Most road-transportation companies own a heterogeneous fleet of vehicles. This diversity in the vehicles capacity might be due to the fact that different customers and locations might require different types of vehicles, e.g.: narrow roads in a city, available parking spaces, vehicle weight restrictions on certain roads, etc. Another reason for owning vehicles with distinct capacities is the natural diversity that arises when vehicle acquisitions are made over time. Accordingly, Ruiz et al. (2004) and Prive et al. (2006) state the importance of developing new vehicle routing methods considering heterogeneous fleets. Some real-life applications of heterogeneous fleets are illustrated in Golden et al. (2002). Examples of these are: urban waste collection, residential pickups and delivery of beverages, food and newspapers delivery, etc.

In the VRP is also common to consider a matrix which elements are the individual costs of travelling from one node to another. These costs can be based on several distance-related factors, such as: actual distance, time employed, fuel consumption, etc. In fact, classical benchmark instances are based on Euclidean distances between each pair of locations, which results in symmetric costs. However, this metric is just a lower bound of the real distance between two nodes connected by a transport network or highway. The real distance will depend upon the specific location of the nodes in the territory and also on the structure of the road network that communicates them. Moreover, when considering oriented networks, real distances might not have to be symmetric Rodríguez and Ruiz (2012).

Several VRP variants have been extensively studied during the last decades (Laporte, 2009). In general, VRP problems are NP-complete within the combinatorial optimisation field. Different VRP variants incorporate different sets of realistic constraints - i.e., vehicle capacity, delivery time windows, service priorities, pickup and delivery options, etc. These constraints make it difficult to find feasible and good solutions in a reasonable computing time. Despite the fact they are common situations in real-life scenarios, the combination of heterogeneous fleets with asymmetric costs has been rarely discussed in the existing literature.

Accordingly, the first goal of this paper is to present an efficient and relatively easy-to-implement methodology for solving both the asymmetric homogeneous vehicle routing problem (AVRP) as well as the asymmetric and heterogeneous vehicle routing problem (AHVRP). Our approach is based on the combination of a randomised version of a classical heuristic with several local search processes specifically adapted to deal with the asymmetric nature of costs. Additionally, the paper also analyses how asymmetric routing costs vary when slight deviations from the homogeneous fleet are considered, i.e., how marginal costs/savings change when a few 'standard' vehicles in the homogeneous scenario are substituted by other vehicles with different loading capacity.

The article is structured as follows: Section 2 contains a mathematical model for the AHVRP. Section 3 reviews the existing related work. Section 4 describes our approach for solving the AVRP as well as the AHVRP. Section 5 presents experimental design. In Section 6 a computational experiment is included for both scenarios. These experiments serve to test the efficiency of our approach and to illustrate its applications in the asymmetric context. In particular, a 'what if' analysis is performed in order to discuss how routing costs can vary when different fleet configurations are considered. Finally, Section 7 points out some future work possibilities and summarises the main results obtained. 


\section{The AHVRP}

This article is focused on a particular variant of the VRP family, called AHVRP. The model used next is an adaptation of the models proposed in Baldacci et al. (2008) and Wen et al. (2010). Both assume symmetric and different transportation costs for each type of vehicle, limited number of vehicles, as well as additional fixed costs for using each type of vehicle.

The model of three sub-indices is formulated as follows. Let $G=(V, A)$ be a complete and directed graph, where $V=\{0,1, \ldots, n\}$ is the set of nodes and $A$ is the set of arcs. The nodes $i=1,2, \ldots, n$ correspond to customers, each with a deterministic demand $d_{i} \geq 0$. The node $i=0$ is the zero-demand depot, i.e., $d_{0}=0$. Let $m>0$ be the number of different types of vehicles. For the $k^{\text {th }}$ type of vehicles $(k=1,2, \ldots, m)$, let $V_{k}=\left\{1,2, \ldots, v_{k}\right\}$ be the set of vehicles of type $k$, with load capacity $Q_{k}>0$. Let $c_{i j}$ be the non-negative distance-based cost associated with the $\operatorname{arc}(i, j) \in A$, and $c_{i i}=+\infty, \forall i \in V$. In the case of calculating the real distances between pairs of locations $(i, j)$, the distance matrix may be asymmetric, i.e., it can happen that $c_{i j} \neq c_{j i}$.

The AHVRP goal is to find the set of minimum-cost roundtrip routes - starting from and ending at the depot - which satisfy all customers' demands, visit each customer only once, and do not exceed the load capacity of each type of vehicle.

$$
\min \sum_{i \in V} \sum_{j \in V} c_{i j} \sum_{k=1}^{m} x_{i j k}
$$

subject to:

$$
\begin{array}{ll}
\sum_{i \in V \backslash\{0\}} x_{i 0 k}=\sum_{j \in V \backslash\{0, i\}} x_{0 j k} & \forall k \in\{1, \ldots, m\} \\
\sum_{k=1}^{m} \sum_{i \in V} x_{i j k}=1 & \forall j \in V \backslash\{0\} \\
\sum_{i \in V \backslash\{h\}} x_{i h k}=\sum_{j \in V \backslash\{h, i\}} x_{h j k} & \forall h \in V \backslash\{0\}, \forall k \in\{1, \ldots, m\} \\
\sum_{j \in V \backslash\{0\}} x_{0 j k} \leq v_{k} & \forall k \in\{1, \ldots, m\} \\
\sum_{i \in V} y_{i j}+d_{j}=\sum_{i \in V} y_{j i} & \forall j \in V \backslash\{0\} \\
0 \leq d_{i} x_{i j k} \leq y_{i j} \leq\left(Q_{k}-j\right) x_{i j k} & \forall(i, j) \in A, \forall k \in\{1, \ldots, m\} \\
x_{i j k} \in\{0,1\} & \forall(i, j) \in A, \forall k \in\{1, \ldots, m\}
\end{array}
$$


In this formulation, for both symmetrical and asymmetrical issues, and for both homogeneous and heterogeneous fleet, $O\left(n^{2} K\right)$ binary variables $x$ are used. The binary variable $x_{i j k}$ in (8) indicates whether or not the arc $(i, j) \in A$ is travelled by a vehicle of type $k(k=1,2, \ldots, m)$. In addition, there are $O(n K)$ variables $y$ where $y_{i j}$ represents the load in the truck arriving at customer $j$ after visiting customer $i$ in terms of units of commodity.

The objective function (1) minimises the total distance-based cost of the arcs used by all $m$ routes generated. Constraint (2) implies that the number of vehicles of each type leaving the depot is the same as the number of vehicles of that type returning to it. Constraints (3) and (4) require that each customer is visited exactly once, and that the same type of vehicle $k$ arrives and leaves each $h$ customer location respectively. Constraint (5) imposes that the number of used vehicles of each type does not exceed the number of available vehicles of that type. Constraint (6) states that the quantity of products $y_{i j}$ in the truck leaving customer $j$ plus the demand of that customer, equals the quantity of products in the truck leaving it after the service has been completed. Constraint (7) guarantees lower and upper bounds ensuring that: the quantity of products $y_{i j}$ in the truck leaving customer $i$ is equals to or greater that its demand, $d_{i}$; and the total demand served by each vehicle $k$ does not exceed the service capacity $Q_{k}$.

\section{Literature review}

As described in Laporte and Nobert (1987) and also in Toth and Vigo (2001), there is a wide range of algorithms for solving the classic CVRP problem-exact algorithms, classical heuristics, and meta-heuristics. However, most techniques have been focused on solving the symmetric CVRP.

It is much less common to find articles that focus exclusively on the asymmetric VRP. In Laporte et al. (1986) an exact algorithm is presented. Fischetti et al. (1994) propose a branch-and-bound algorithm and its practical application to a real case of pharmaceutical distribution in a city of Italy. In Vigo (1996), the author discusses the extension to the AVRP of two of the most important and successful techniques: the savings algorithm of Clarke and Wright (1964), and the optimisation method of Fisher and Jaikumar (1981). The author states that the solutions found using the proposed asymmetric version of the CWS quickly evolves to worse values as the number of customers increases, in addition to the inconvenience of the parameter combination for the parametric saving function. More recently, there are two promising techniques that have been shown to work well in both cases of symmetrical and asymmetrical CVRP. The first is a general heuristic proposed by Pisinger and Røpke (2007) which is the result of a unified heuristic for several variants of VRP using the adaptive large neighbourhood search (ALNS). The second is a memetic algorithm (MA) described in Nagata (2007). These algorithms have been selected by their performance and recognition. We have strived for a balance between simple classical techniques and also current and state-of-the-art methods. 
Many combinatorial problems consider more than one fleet of vehicles with different capacities. Since the first axe of our addressed rich problem is the asymmetric costs matrix, the second axe is the heterogeneous fleet vehicle routing problem (HVRP). The HVRP has been quite studied in the literature. For instance, Baldacci et al. (2008) present a comprehensive description of some of its most important variants. On this last study, we can appreciate how the research community has addressed the HVRP in different ways. First, considering either a limited or an unlimited number of available vehicles of each type. Second, considering fixed and/or variable costs associated with the use of each type of vehicle. Each vehicle could have a fixed cost for using it in a trip (i.e., $\left.F_{k} \neq 0 / \forall i, j, k\right)$ as well as a variable cost that is the result of multiplying a coefficient by the distance of the assigned route (i.e., $c_{i j k_{1}} \neq c_{i j k_{2}} / \forall k$ ). These costs used to be associated to each type of vehicle. The combinations of the aforementioned parameters have created the main HVRP families, known as:

- H1: fleet size and mix VRP with fixed and variable costs where an unlimited number of vehicles is considered for minimising the addition of using a specific vehicle and the variable distance.

- H2: fleet size and mix VRP with only fixed costs where an unlimited number of vehicles is considered for minimising fixed cost of all used vehicles.

- H3: fleet size and mix VRP with only variable costs where an unlimited number of vehicles is considered for minimising the variable distance of all routes.

- H4: heterogeneous fixed fleet VRP with only variable costs where a limited number of vehicles is used to minimising the variable cost.

- H5: heterogeneous fixed fleet VRP with fixed and variable costs where a limited number of vehicles is used to minimising both variable and fixed costs.

Notice that there are others HVRP branches considering constraints like site-dependent, site-road, etc. For instance, Prins (2002) considers that each vehicle can optionally perform several trips using a savings-based heuristic but without any extra cost. The basic family $(\mathrm{H} 0)$ consists in the natural condition of considering a heterogeneous fleet inside of the combinatorial problem.

The rich vehicle routing problem (RVRP) is a generalised variant of the VRP where several constraints, aspects or objectives functions are considered at the same time. Examples of constraints and assumptions considered in RVRPs could be: multi-depot, periodic visits to clients, open routes, multi-products, time windows, etc. Drexl (2012) compiles some current needs on the RVRP field as well as a state-of-the-art of scientific research and commercial software. In some studies like Prins (2002), Bolduc et al. (2006), Irnich (2008), Rieck and Zimmermann (2010), Oppen et al. (2010), Prescott-Gagnon et al. (2012) and Vallejo et al. (2012) the variable and fixed costs are 
ignored when combined with other routing features. In fact, Bolduc et al. (2006) is included with other papers in a Special Issue on Rich Vehicle Routing Problems (Hartl et al., 2006). Table 1 summarises the work done in several types of HVRP. On its columns, we can appreciate the addressed HVRP families, the applied methodology, as well as others routing constraints considered on each study. Also, the first part of the table presents papers exclusively dedicated to the HVRP, while the second part shows papers related to RVRP where the HVRP is also considered together with other constraints. Most of these works just include the heterogeneous capacity of vehicles (H0) and not the related costs associated with each type of vehicle. Therefore, there is not a single way to include the HVRP feature in a RVRP.

Table 1 Summary of published HVRP studies

\begin{tabular}{|c|c|c|c|}
\hline Study & HVRP family & Method & Extra constraints \\
\hline Golden et al. (1984) & $\mathrm{H} 0, \mathrm{H} 2$ & Heuristics & \\
\hline Taillard (1999) & $\mathrm{H} 0, \mathrm{~F} 2, \mathrm{H} 4$ & $\begin{array}{c}\text { Column generation-based } \\
\text { heuristic }\end{array}$ & \\
\hline Gendreau et al. (1999) & $\mathrm{H} 0, \mathrm{H} 2, \mathrm{H} 3$ & Tabu search & \\
\hline $\begin{array}{l}\text { Wassan and } \\
\text { Osman (2002) }\end{array}$ & $\mathrm{H} 0, \mathrm{H} 2, \mathrm{H} 3$ & Tabu search & \\
\hline $\begin{array}{l}\text { Renaud and } \\
\text { Boctor (2002) }\end{array}$ & $\mathrm{H} 0, \mathrm{H} 2$ & Heuristic-based & \\
\hline Lima et al. (2004) & $\mathrm{H} 0, \mathrm{H} 2$ & MA & \\
\hline Tarantilis et al. (2004) & $\mathrm{H} 0, \mathrm{H} 4$ & $\begin{array}{l}\text { Threshold accepting } \\
\text { algorithms }\end{array}$ & \\
\hline Choi and Tcha (2007) & $\begin{array}{l}\mathrm{H} 0, \mathrm{H} 1, \\
\mathrm{H} 2, \mathrm{H} 3\end{array}$ & Branch-and-bound & \\
\hline Li et al. (2007) & $\mathrm{H} 0, \mathrm{H} 4$ & Record-to-record & \\
\hline Prins (2009) & $\begin{array}{l}\mathrm{H} 0, \mathrm{H} 1, \mathrm{H} 2, \\
\mathrm{H} 3, \mathrm{H} 4\end{array}$ & MA & \\
\hline Imran et al. (2009) & $\mathrm{H} 0, \mathrm{H} 1, \mathrm{H} 3$ & $\begin{array}{l}\text { Variable neighbourhood- } \\
\text { based heuristic }\end{array}$ & \\
\hline Liu et al. (2009) & $\mathrm{H} 0, \mathrm{H} 2$ & Genetic algorithm & \\
\hline Brandao (2009) & $\mathrm{H} 0, \mathrm{H} 2, \mathrm{H} 3$ & Tabu search & \\
\hline $\begin{array}{l}\text { Euchi and } \\
\text { Chabchoub (2010) }\end{array}$ & $\mathrm{H} 0, \mathrm{H} 4$ & Hybrid tabu search & \\
\hline Li et al. (2010) & $\mathrm{H} 0, \mathrm{H} 5$ & $\begin{array}{l}\text { Adaptive memory } \\
\text { programming } \\
\text { metaheuristic }\end{array}$ & \\
\hline Brandao (2011) & $\mathrm{H} 0, \mathrm{H} 3, \mathrm{H} 4$ & Tabu search & \\
\hline Penna et al. (2013) & $\begin{array}{l}\mathrm{H} 0, \mathrm{H} 1, \mathrm{H} 2, \\
\mathrm{H} 3, \mathrm{H} 4, \mathrm{H} 5\end{array}$ & $\begin{array}{c}\text { Iterative local } \\
\text { search with variable } \\
\text { neighbourhood descent }\end{array}$ & \\
\hline $\begin{array}{l}\text { Subramanian } \\
\text { et al. (2012) }\end{array}$ & $\begin{array}{l}\mathrm{H} 0, \mathrm{H} 1, \mathrm{H} 2, \\
\mathrm{H} 3, \mathrm{H} 4, \mathrm{H} 5\end{array}$ & $\begin{array}{l}\text { Iterative local search with } \\
\text { set partitioning }\end{array}$ & \\
\hline
\end{tabular}


Table 1 Summary of published HVRP studies (continued)

\begin{tabular}{|c|c|c|c|}
\hline Study & HVRP family & Method & Extra constraints \\
\hline Bolduc et al. (2006) & $\mathrm{H} 0$ & Heuristics & Multi-period \\
\hline Irnich (2008) & $\mathrm{H} 0$ & $\begin{array}{l}\text { Local search-based } \\
\text { metaheuristic }\end{array}$ & $\begin{array}{l}\text { Multi-depots, and } \\
\text { time windows }\end{array}$ \\
\hline $\begin{array}{l}\text { Baldacci and } \\
\text { Mingozzi (2009) }\end{array}$ & H0, H5 & $\begin{array}{l}\text { MIP model with set } \\
\text { partitioning }\end{array}$ & $\begin{array}{l}\text { Site-dependent, and } \\
\text { multi-depots }\end{array}$ \\
\hline $\begin{array}{l}\text { Rieck and } \\
\text { Zimmermann (2010) }\end{array}$ & H0 & MILP model & $\begin{array}{l}\text { Time windows, } \\
\text { and simultaneous } \\
\text { delivery and pickup }\end{array}$ \\
\hline Oppen et al. (2010) & $\mathrm{H} 0$ & Column generation-based & $\begin{array}{l}\text { Multi-products, multiple } \\
\text { trips, precedence and } \\
\text { inventory constraints }\end{array}$ \\
\hline $\begin{array}{l}\text { Prescott-Gagnon et al. } \\
(2012)\end{array}$ & H0 & Heuristics & $\begin{array}{l}\text { Multi-depots, intra-route } \\
\text { replenishments, time } \\
\text { windows, driver shifts and } \\
\text { optional customers }\end{array}$ \\
\hline Vallejo et al. (2012) & $\mathrm{HO}$ & $\begin{array}{l}\text { Memory-based approach } \\
\text { with clustering }\end{array}$ & $\begin{array}{l}\text { Time windows, multi- } \\
\text { depots, and multi-trip }\end{array}$ \\
\hline
\end{tabular}

To the best of our knowledge, there is not any published work on the AHVRP. The most approximated ones are presented in Marmion et al. (2010) and Pessoa et al. (2008). In the first study, the authors analyse the sensitivity of two classical neighbourhoods methods for the AHVRP. Thus, they simulate a heterogeneous fleet assigning different variable costs to each vehicle, but the capacity remains unchanged. On Pessoa et al. (2008), the authors developed a set of robust branch-cut-and-price algorithms for several VRPs. Some promising experiments are presented, but changes in the capacity of fleets are not justified for the HVRP. More details about these two studies are discussed later.

\section{Our approach for the AHVRP}

Our approach focuses on solving two realistic problems: the AVRP as well as the AHVRP.

\subsection{Constructive component}

The algorithm we propose is based on a randomised version of the Clark and Wright savings (CWS) heuristic (Clarke and Wright, 1964). The CWS is one of the most commonly cited methods in the VRP literature. It uses the concept of savings associated with each arc for merging routes. At each step, the arc with the greatest savings is selected if and only if the two corresponding routes can be combined into a new feasible route and if the selected arc is composed of nodes that are directly connected with the depot. We address the AVRP and AHVRP without considering an extensive asymmetric saving list - i.e., a list including two directed arcs for each pair of customers. Instead we consider a weighted savings list considering just one arc for each pair of customers. Also, we consider the direction of the resulting route after each merging. 
Figure 1 Flowchart of the asymmetric SR-GCWS for heterogeneous fleets (see online version for colours)

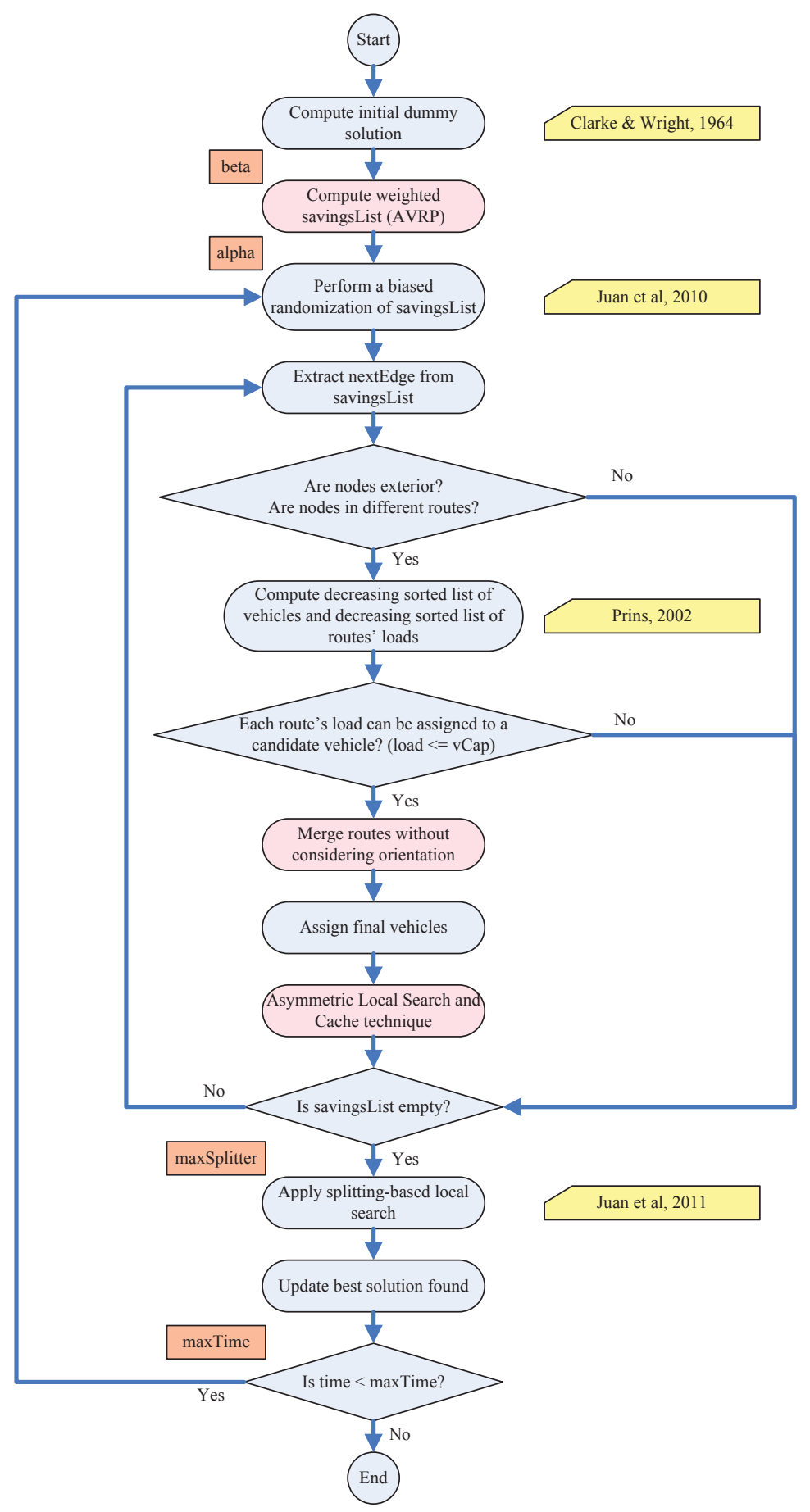


Figure 1 shows a flowchart diagram offering a high-level view of our algorithm. Our approach starts solving the problem as proposed in the CWS heuristic - i.e.: computing a dummy solution assigning one round-trip route from the depot to each customer. Then the algorithm computes the weighted savings list using an auxiliary parameter $\beta$ (see below the formulas for weighted saving, i.e., $\hat{S}_{i j}$, and CWS saving, i.e., $S_{i j}$ ). At this point the CWS heuristics is combined with a randomisation process. We use a pseudo-geometric distribution to assign a selection probability to each edge in the savings list (alpha). Moreover, this selection probability is coherent with the weighted saving value associated with each edge, i.e., edges with higher savings will be more likely to be selected from the list than those with lower savings. Therefore, each combination of edges has a chance of being selected and merged with previously built routes. Then, a multi-start process is initiated and controlled by a time parameter (maxTime). At each iteration of this process, different edges are selected using the aforementioned biased probability distribution. This allows obtaining different outputs at each iteration. After merging, we improve the merged route applying two promising local search processes. At the end, we apply a general local search to the whole solution which is explained in the next section.

The validation of the capacity constraint in a heterogeneous fleet is addressed as a vehicle-trip assignment. For this, an effective method based on CWS is proposed in Prins (2002). The list of vehicles and the list of routes are sorted decreasingly by capacity and accumulated demands respectively; after that, a temporary assignment between the two lists is searched. If a successful match - including all previously routes plus the new merged one - is found, then the capacity constraint is satisfied and the temporary assignment becomes final. Otherwise, the merge becomes unfeasible. If a situation arises in which the number of routes is greater than the number of vehicles, then new fictitious vehicles are assigned to the remaining routes. Notice that this vehicle assignment validation is made for each possible saving, increasing the computational operations. The author also imposes an assumption that the largest demand cannot exceed the capacity of the smallest vehicle.

One important contribution of our approach is the fact that we consider a weighted savings list merging two routes without taking into account directions at this initial stage. See an example in Figure 2. The application of a local search will help to define the best direction. The weighted saving associated with an arc connecting customers $i$ and $j$ is defined as:

$$
\hat{S}_{i j}=\beta * \max \left\{S_{i j}, S_{j i}\right\}+(1-\beta) * \min \left\{S_{i j}, S_{j i}\right\}
$$

where $\beta \in[0.5,1]$ and $S_{i j}=c_{0 i}+c_{0 j}-c_{i j}$.

The disregard of orientation is important given that an asymmetric savings list does not choose arcs which do not match their orientation established. For example, it rejects the routes in Figure 2 since they have different orientation. This reduces the solution space and worsens the general solution; even some obtained solutions are using a greater number of vehicles. 
Figure 2 Merging routes $R_{1}=\{0, h, i, 0\}$ and $R_{2}=\{0, k, j, 0\}$ with different orientation into a route $R=\{0, h, i, j, k, 0\}$
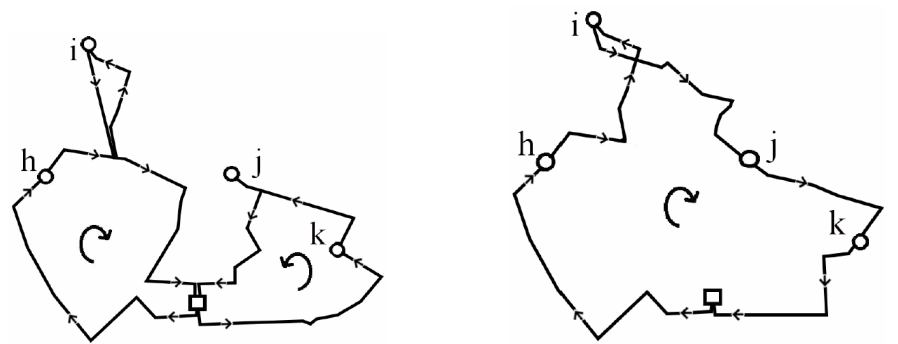

Note: The route $R_{2}$ is reversed and some edges are changed given that it is an urban area with some one-way streets.

Given that the orientations are not considered, an original local search for the asymmetric context was created exploring the near solution space with few steps. It represents another important contribution of our approach.

\subsection{Asymmetric local searches}

Once a merged route is obtained, two local searches are applied in order to explore the solution space with few steps. The first local search procedure is the so called reversing routes local search. This procedure intends to find an improvement in the order and orientation of the nodes. Given a merged route, we first try to sort the nodes in a more efficient way. If a route is composed by more than four nodes, then we take each four nodes - i.e., $(i, j, k, 1)$ - and try to determine if a swapping of two middle-nodes could improve the cost - i.e., $(i, k, j, l)$. After that, we try to reverse the order in which nodes are traversed.

A second local search, originally described in Juan et al. (2011), is focused on checking if a given set of nodes already exists in a memory but with a better order of the nodes. The basic idea of this learning mechanism is to store in a cache memory the best-know order to travel among the nodes that constitute one route. This cache is constantly updated whenever a better order with a lower cost is found for a given set of nodes. At the same time, the routes contained in this cache are re-used whenever possible to improve newly merged routes. Notice that this procedure does not search a new vehicle assignment. The previously assigned vehicle to each route remains unchanged during this process.

Finally, once all edges in the saving list have been considered, the resulting solution is improved through a Splitting local search method proposed in Juan et al. (2011). The current solution is divided into disjoint subsets of routes together with their previously assigned vehicles; then, each of these subsets are solved applying the same methodology described before during a given number of iterations (maxSplitter). This tries to apply a 'divide and conquer' approach since smaller instances could be easier to solve. So a new set of routes could be created on each partition with the previously assigned vehicles. 


\section{Experimental design}

The most commonly-used methodology to compare the performance of different algorithms for solving VRPs consists in running these algorithms over a set of well-defined benchmark instances. In the case of the CVRP or the AVRP, several benchmark sets are available through open-access websites, so that researchers worldwide can use them. Usually, these datasets contain complete information, including not just the instance inputs and the best-known value for the objective function, but also a complete description of the corresponding solution - i.e., the specific composition of each route in the best-known solution. In the case of the AHVRP, however, there is not a commonly-accepted set of instances to test algorithms, since the AHVRP has rarely been discussed so far in the literature.

For the AVRP, some researchers have used a set of real instances related to Fischetti et al. (1994). In our case, we have selected 20 public AVRP instances from http://soa. iti.es/files/Instances_CVRP.7z. Details on these instances can be found in Rodríguez and Ruiz (2012). They have been generated with a realistic perspective and mathematical justification. The selection was made at random among the set of medium and large-size instances (in terms of number of nodes). They have 50 or 100 customers and are designed to employ a homogeneous fleet with the number of vehicles ranging from 2 to 7 . These instances consider large demands and vehicle capacities, as well as random location of the nodes within intra-city areas. The depot may be in the centre of the area, those with ' $\mathrm{C}$ ' in the second letter of the name, or a random position, those with 'A'. The intra-city instances were chosen given that they represent a higher asymmetry degree. Rodríguez and Ruiz (2012) conclude that these instances affect in a statistically significant way the CPU time needed by some algorithms and deteriorate the quality of the solutions obtained.

We have selected the following state-of-the-art AVRP methods in order to compare with:

- General heuristic of Pisinger and Røpke (2007). It is a unified heuristic that works for several variants of routing problems and that uses an ALNS.

- MA of Nagata (2007). Similar to ALNS, MA is a very powerful and recent AVRP metaheuristic.

The previous algorithms have been selected by their performance and recognition. We have strived for a balance between simple classical techniques and current and state-of-the-art methods. Algorithms MA and ALNS were run from the original code which was kindly provided by their respective authors. No code modification was carried out and the methods were run according to their recommendations.

For the AHVRP, Fischetti et al. (1994) developed some preliminary experiments based on a set of real AVRP instances. Likewise, Marmion et al. (2010) analysed the use of a heterogeneous fleet. However, these studies have only considered the effect of variable cost on vehicles selection, but no differences in actual vehicle capacities are considered. Also Pessoa et al. (2008) have used these benchmarks first modifying the capacity of the original fleets and then running the experiments with homogeneous fleets.

Therefore, we propose to use exactly the same nodes as in these AVRP instances, including their asymmetric costs and demands, and the same number of vehicles. We then consider a heterogeneous fleet composed of 'standard' vehicles - i.e., vehicles with 
the capacity defined in the AVRP instances - and 'non-standard' vehicles with modified capacities. In our opinion, this is a natural way to adapt the homogeneous-capacity instances, since it allows the decision-maker to answer sensitivity-analysis questions such as: "How would my routing costs be changed if we could employ one or two trucks with a different capacity?".

Thus, in order to test our approach, a total of twenty classical AVRP instances were selected and adapted as 'base' AHVRP instances. For each base instance, six different fleet typologies were defined - thus, 120 different instances were considered in total. These fleet typologies are partially composed of 'standard' vehicles, each of them with capacity $Q_{0}$, but they differ in their exact composition as explained in the following general rule:

- fleet 150-125: two 'standard' trucks are substituted by a 'large' truck (with capacity $Q_{1}=150 \% \cdot Q_{0}$ ) and by a 'large-medium' truck (with capacity $Q_{l m}=125 \% \cdot Q_{0}$ ), respectively

- fleet 125-125: two 'standard' trucks are substituted by two 'large-medium' trucks

- fleet 125-80: two 'standard' trucks are substituted by a 'large-medium' truck and by a 'small' truck (with capacity $Q_{s}=80 \% \cdot Q_{0}$ ), respectively

- fleet 100-100: the homogeneous case where all trucks are 'standard'

- fleet 90-90: two 'standard' trucks are substituted by two 'small-medium' trucks (with capacity $Q_{s m}=90 \% \cdot Q_{0}$ )

- fleet 90-80: two 'standard' trucks are substituted by a 'small-medium' truck and by a 'small' truck, respectively.

Notice, however, that in some cases a reduction in the fleet capacity might cause the infeasibility of the problem, i.e., the total demand to be satisfied might be greater than the total fleet capacity. In those particular cases, an additional 'standard' vehicle is added to the fleet to promote feasibility of the problem.

\section{Experimental results}

In order to validate our algorithm, we first present the results of an AVRP homogeneous case. For this, we compare our results with those obtained using the MA of Nagata (2007) and the ALNS of Pisinger and Røpke (2007). Finally, we developed some experiments for the AHVRP.

The algorithm described in this paper has been implemented as a Java application. An Intel QuadCore i5 at 3.2 GHz and 4 GB RAM was used to perform all tests, which were run over Windows XP.

\subsection{Homogeneous instances}

For the 20 AVRP instances, we have used ten random seeds (ten replicas), an elapsed time of 1 minute (maxTime) for each seed, and 60 iterations for the splitting technique (maxSplitter). In order to perform a biased randomisation of the weighted savings list, a 
quasi-geometric distribution with a parameter $\alpha$ randomly selected in the interval $[0.5,0.10]$ was used; and the value chosen for the weighted saving was $\beta=0.6$.

The Nagata algorithm was executed with a parameter setting: $N_{p o p}=100, N_{c h}=30$, ten trials and two parents. Also ten runs with elapsed time of 1 minute were executed for each instance. For the ALNS, only one run was executed for each instance without time limit. Both algorithms were run from the original $\mathrm{C}++$ code which was kindly provided by their respective authors. No code modification was carried out and the methods were run according to their recommendations.

The results of these tests are summarised in Table 2, which contains the following information for each instance: name of instance; number of nodes; number of vehicles; the best solution of ten replicas of the MA, $\{1\}$; the ALNS solution, $\{2\}$; gap, expressed as a percentage value, between $\{1\}$ and $\{2\}$; the time used for ALNS in seconds; our best solution found, OBS $\{3\}$; and gap between $\{1\}$ and $\{3\}$.

Table 2 Comparison of results for AVRP instances

\begin{tabular}{lcccccccc}
\hline Instance & \# nodes & \# vehicles & $\begin{array}{c}\text { MA } \\
\{1\}\end{array}$ & $\begin{array}{c}\text { ALNS } \\
\{2\}\end{array}$ & $\begin{array}{c}\text { Gap } \\
\{1\}-\{2\}\end{array}$ & Time $(s)$ & $\begin{array}{c}\text { OBS } \\
\{3\}\end{array}$ & $\begin{array}{c}\text { Gap } \\
\{1\}-\{3\}\end{array}$ \\
\hline G-A-CAA0501 & 50 & 2 & 370.26 & 370.26 & $0.00 \%$ & 17.83 & 370.26 & $0.00 \%$ \\
G-A-CAA0502 & 50 & 3 & 414.44 & 414.44 & $0.00 \%$ & 13.30 & 414.44 & $0.00 \%$ \\
G-A-CAA0503 & 50 & 4 & 444.69 & 444.69 & $0.00 \%$ & 10.48 & 444.69 & $0.00 \%$ \\
G-A-CAA0504 & 50 & 2 & 362.01 & 362.01 & $0.00 \%$ & 17.95 & 362.01 & $0.00 \%$ \\
G-A-CAA0505 & 50 & 3 & 395.78 & 395.78 & $0.00 \%$ & 15.59 & 398.47 & $0.68 \%$ \\
G-A-CAA1001 & 100 & 5 & 661.88 & 664.53 & $0.40 \%$ & 43.03 & 675.31 & $2.03 \%$ \\
G-A-CAA1002 & 100 & 5 & 621.06 & 622.67 & $0.26 \%$ & 39.36 & 625.82 & $0.77 \%$ \\
G-A-CAA1003 & 100 & 5 & 627.29 & 627.29 & $0.00 \%$ & 42.23 & 627.29 & $0.00 \%$ \\
G-A-CAA1004 & 100 & 6 & 681.89 & 681.89 & $0.00 \%$ & 34.84 & 686.25 & $0.64 \%$ \\
G-A-CAA1005 & 100 & 7 & 810.97 & 810.97 & $0.00 \%$ & 29.03 & 820.56 & $1.18 \%$ \\
G-C-CAA0501 & 50 & 2 & 376.62 & 376.62 & $0.00 \%$ & 17.70 & 376.62 & $0.00 \%$ \\
G-C-CAA0502 & 50 & 3 & 372.48 & 372.48 & $0.00 \%$ & 13.31 & 372.48 & $0.00 \%$ \\
G-C-CAA0503 & 50 & 4 & 404.30 & 404.30 & $0.00 \%$ & 10.36 & 404.30 & $0.00 \%$ \\
G-C-CAA0504 & 50 & 2 & 361.74 & 361.74 & $0.00 \%$ & 17.84 & 361.74 & $0.00 \%$ \\
G-C-CAA0505 & 50 & 3 & 386.73 & 386.73 & $0.00 \%$ & 13.80 & 386.73 & $0.00 \%$ \\
G-C-CAA1001 & 100 & 5 & 596.54 & 596.86 & $0.05 \%$ & 40.83 & 600.35 & $0.64 \%$ \\
G-C-CAA1002 & 100 & 5 & 578.15 & 578.15 & $0.00 \%$ & 38.61 & 583.39 & $0.90 \%$ \\
G-C-CAA1003 & 100 & 5 & 561.08 & 561.08 & $0.00 \%$ & 41.13 & 566.10 & $0.89 \%$ \\
G-C-CAA1004 & 100 & 6 & 660.81 & 660.81 & $0.00 \%$ & 35.19 & 664.14 & $0.50 \%$ \\
G-C-CAA1005 & 100 & 7 & 652.08 & 652.18 & $0.02 \%$ & 28.63 & 652.42 & $0.05 \%$ \\
\hline & & & & & $0.04 \%$ & & & $0.41 \%$ \\
\hline
\end{tabular}

Notice that the results obtained with our approach are quite competitive, showing an average gap of $0.41 \%$ with respect to Nagata (2007), which always obtains the best results. Our approach also found exactly the same solution (gap $=0.00 \%$ ) for 10 out of 20 instances. Notice that all three algorithms were run using the same computing time and machine. The only difference is that our algorithm was codified in Java, which runs over a virtual machine and thus has a lower performance than a code implemented in native $\mathrm{C} / \mathrm{C}++$. 
Table 3 Experimental results for small-size instances with different fleet configurations

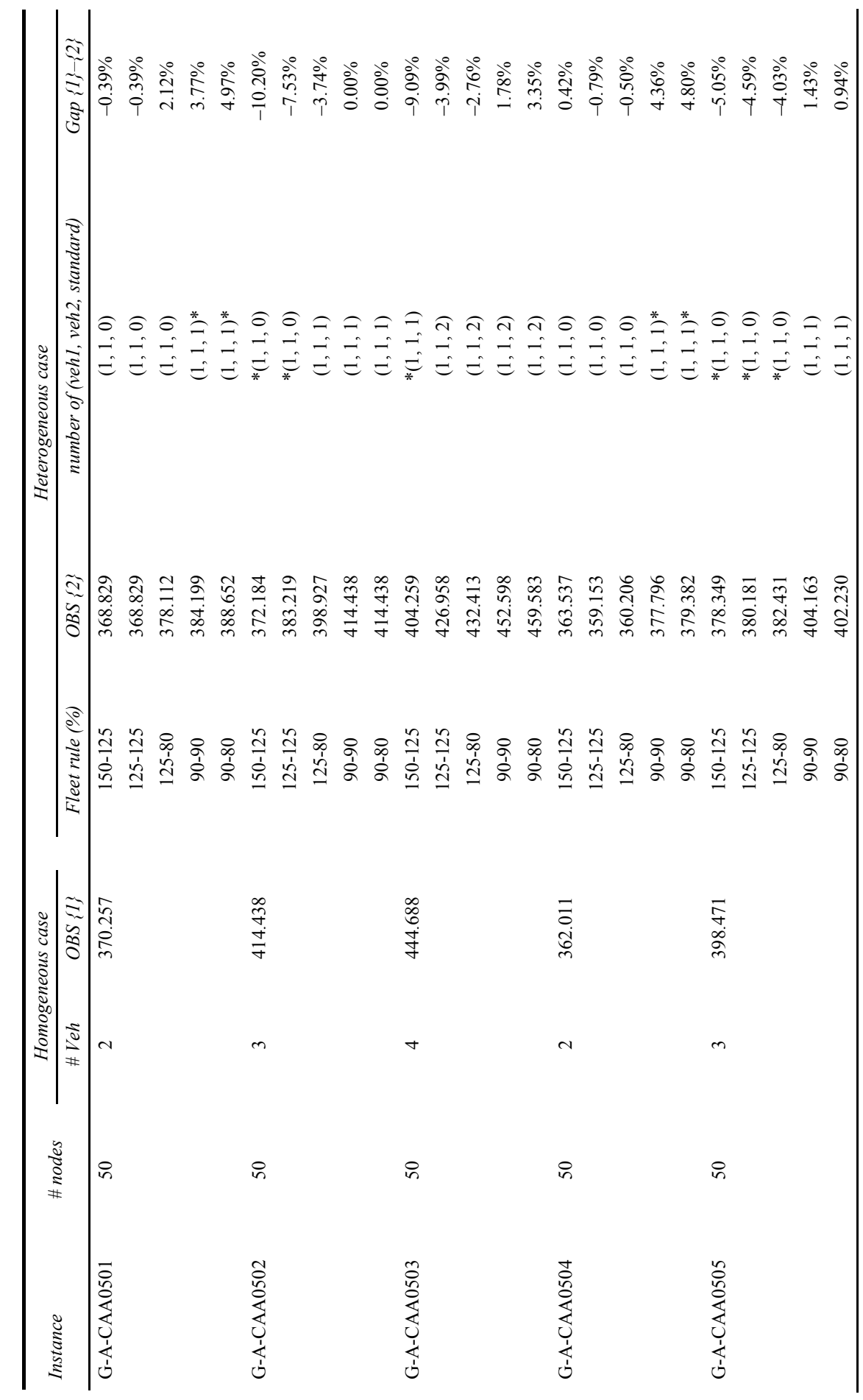


Table 3 Experimental results for small-size instances with different fleet configurations (continued)

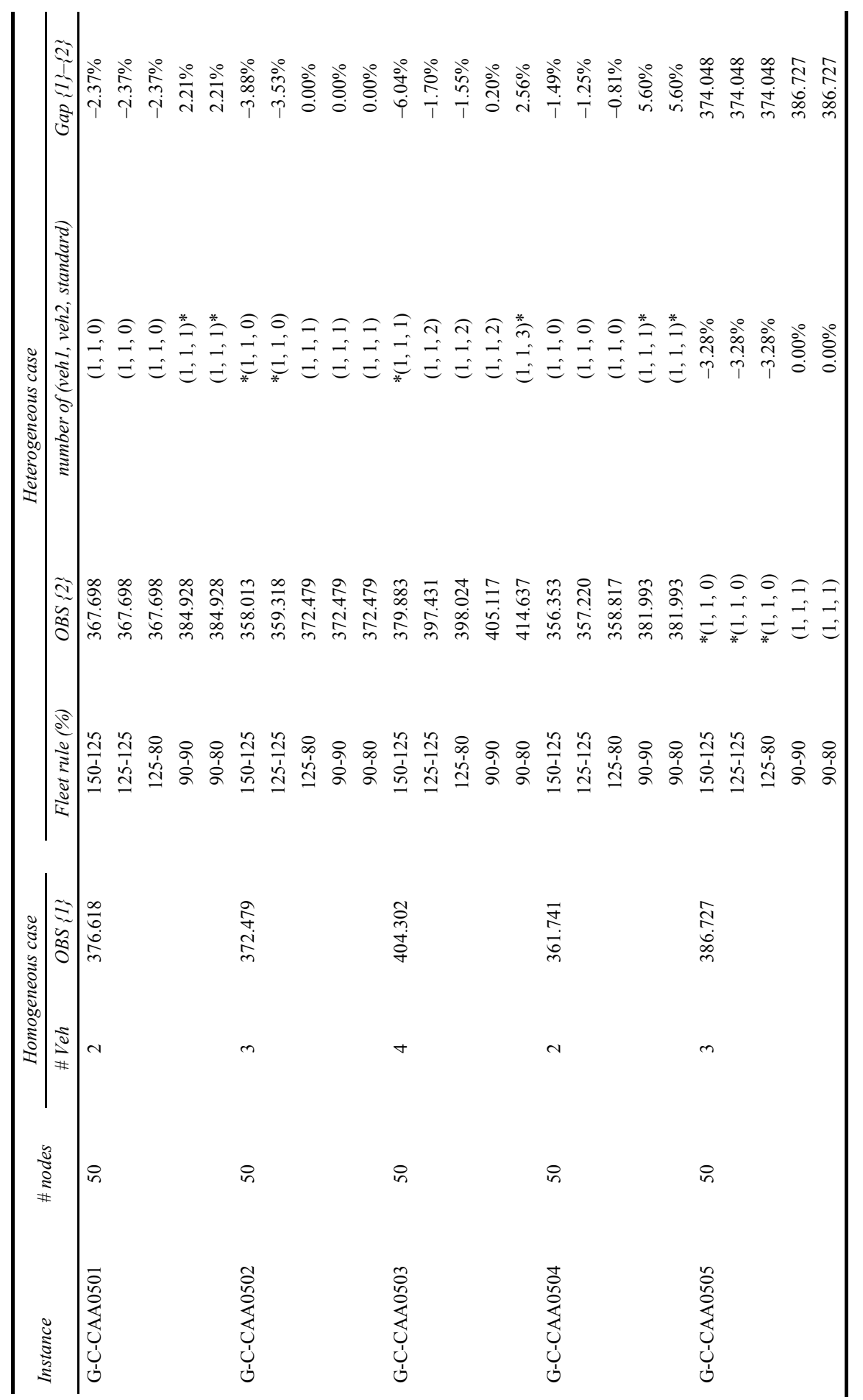


Table 4 Experimental results for medium-size instances with different fleet configurations

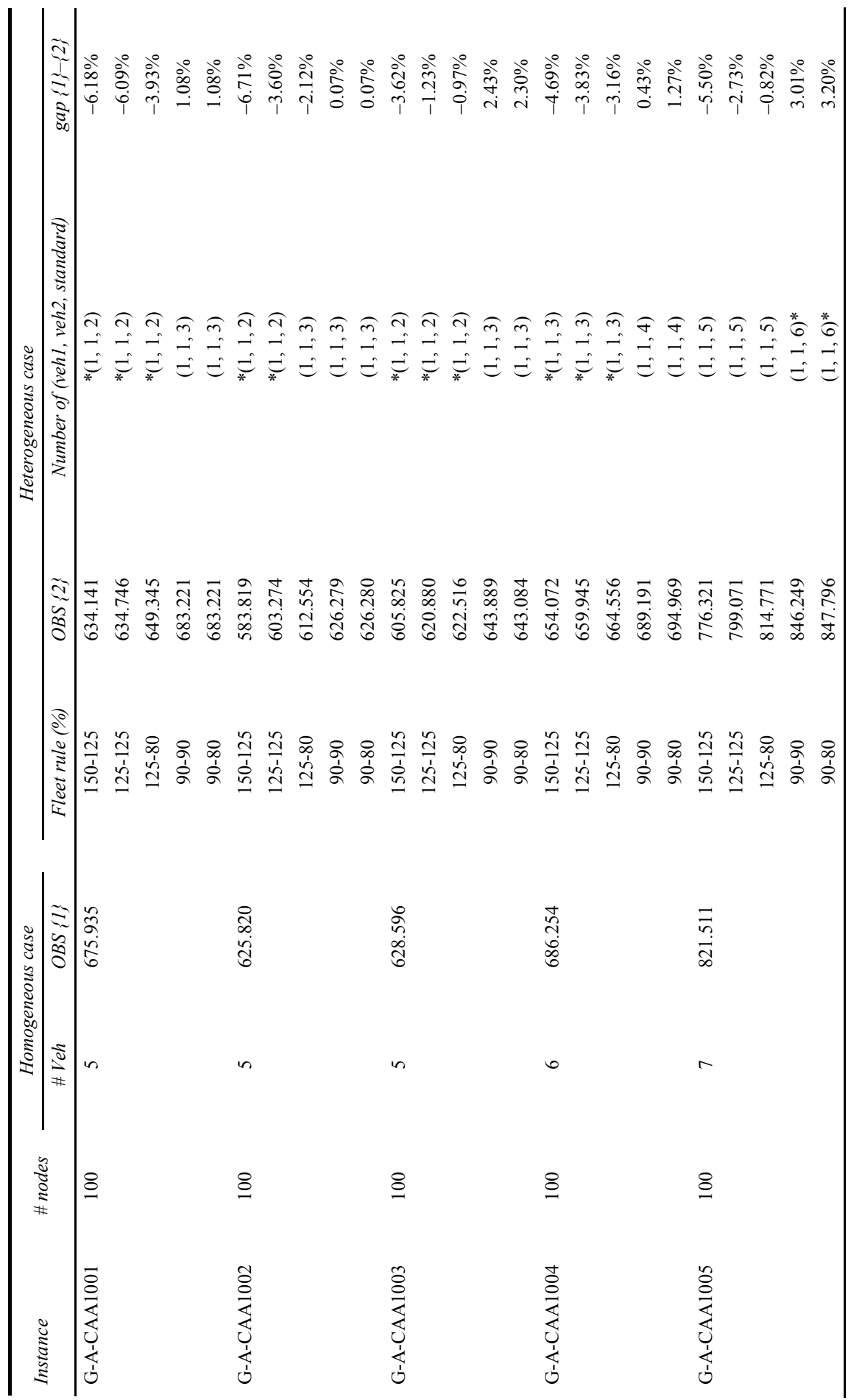


Table 4 Experimental results for medium-size instances with different fleet configurations (continued)

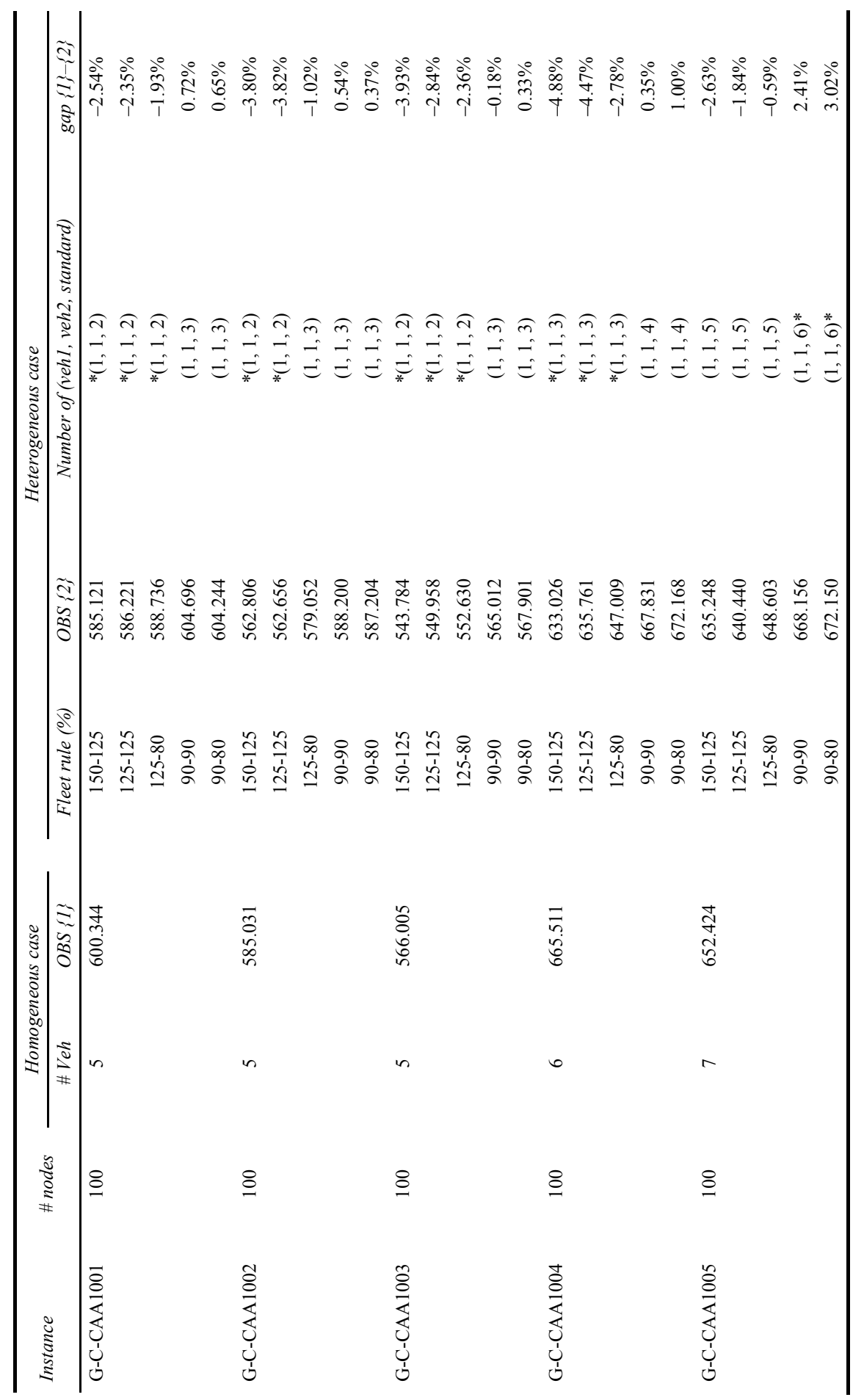




\subsection{Analysing different fleet compositions}

Once the proposed approach has shown to be competitive for the AVRP, the next logical step is to use it for solving the AHVRP. Thus, twenty AVRP instances were adapted as 'base' AHVRP instances. For each base instance, six different fleet typologies were defined, see Section 5 for more details. Thus, 120 different instances were considered in total. For each of them, ten random seeds (ten replicas) and an elapsed time of 1 minute were used.

Tables 3 and 4 contain, for each base instance, the following information: name of instance; number of nodes; number of vehicles; our best solution found for the homogeneous case, OBS $\{1\}$; different fleet rules for the heterogeneous case, each of them defining a new routing instance; our best solution found for the heterogeneous case, OBS $\{3\}$; the obtained number of vehicles for the fleet configuration; and percentage gap between the BKS for the homogeneous case and the OBS for the heterogeneous case. Instances are distributed in both tables according to their sizes.

Observe that * highlights different number of vehicles. For example, $(1,1,1)^{*}$ of the fifth row remarks that this heterogeneous solution is using one more vehicle than the homogeneous solution. It uses one vehicle of $90 \%$ of capacity, one vehicle of $80 \%$ and one 'standard' vehicle. Instead, $*(1,1,0)$ of the sixth row remarks that this solution is using one less 'standard' vehicle.

Figure 3 Surface plot of average gap vs. fleet configuration (see online version for colours)

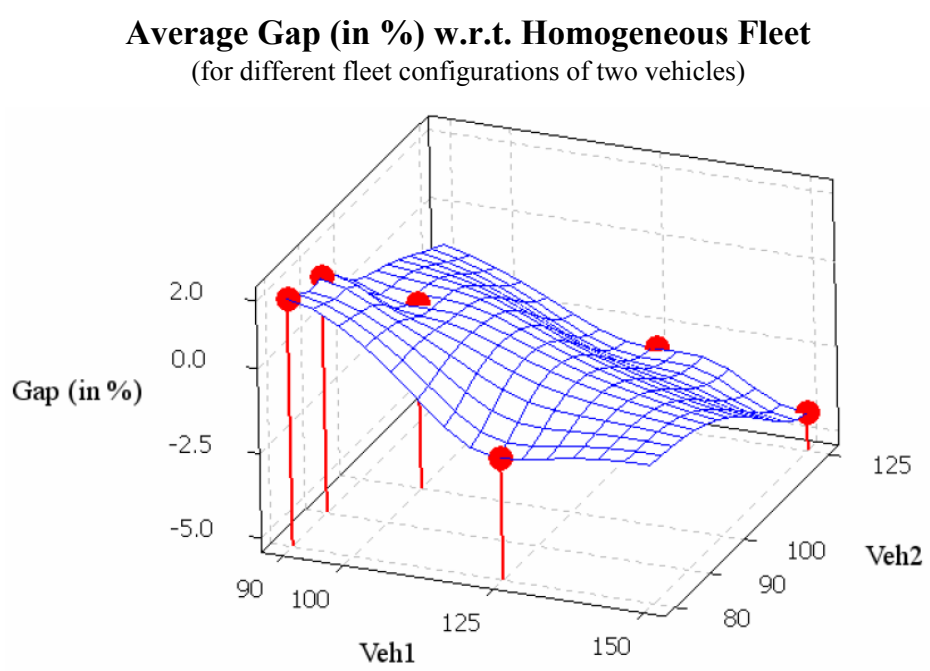

Figure 3 shows a 3D scatter plot representing the average gap associated with each of the six fleet configurations considered in this article. In other words, for each fleet rule, the twenty gaps with respect to the homogeneous OBS - one per base instance - have been averaged. From these results, it can be noticed the following:

- just by employing two large vehicles (fleet 150-125) instead of two 'standard' vehicles (fleet 100-100), it is possible to obtain noticeable costs reductions that can go up to $10 \%$ in some instances (e.g., G-A-CAA0502) 
- likewise, when using two small vehicles (fleet 90-80) instead of two 'standard' vehicles, costs can suffer an increase of about $5 \%$ for some instances (e.g., G-CCAA0504).

Therefore, it can be concluded that routing costs can be in fact quite sensitive to small variations in the fleet configuration. This justifies the necessity for employing new approaches in real-life routing applications, i.e., algorithms which are able to deal with both asymmetric costs as well as heterogeneous fleets.

\section{Conclusions}

In this paper, we have discussed the importance of taking into account both heterogeneous fleets and asymmetric costs in realistic VRPs. Despite the fact that most real-life fleets of vehicles are heterogeneous and that real-life distances are frequently asymmetric - especially in urban transportation -, there is a lack of works considering both situations simultaneously. Accordingly, we have presented a hybrid algorithm for solving the AHVRP. This algorithm combines a randomised savings heuristic with three local search processes specifically adapted to the asymmetric nature of costs in real-life scenarios.

A complete set of AVRP tests have been performed to illustrate the methodology and analyse its efficiency when compared with two state-of-the-art algorithms. The results show that our approach is able to produce competitive results for the AVRP while, at the same time, it is much simpler to implement and requires less parameters - and fine-tuning efforts - than current state-of-the-art algorithms.

Moreover, since our methodology can also consider heterogeneous fleets, a set of benchmarks for the AHVRP have been developed and a sensitivity analysis on the fleet composition has been performed. This last experiment shows how decision-makers can benefit from our approach when deciding the actual composition of their heterogeneous fleets.

\section{Acknowledgements}

This work has been partially supported by the Ibero-American Program for Science, Technology and Development (CYTED2010-511RT0419, IN3-HAROSA network) and by the Spanish Ministry of Science and Innovation (TRA2010-21644-C03).

\section{References}

Baldacci, R. and Mingozzi, A. (2009) 'A unified exact method for solving different classes of vehicle routing problems', Mathematical Programming, Vol. 120, No. 2, pp.347-380.

Baldacci, R., Battarra, M. and Vigo, D. (2008) 'Routing a heterogeneous fleet of vehicles', in Golden, B., Raghavan, S. and Wasil, E. (Eds.): The Vehicle Routing Problem: Latest Advances and New Challenges, Vol. 43 of Operations Research/Computer Science Interfaces, pp.3-27, Springer, USA.

Bolduc, M-C., Renaud, J. and Montreuil, B. (2006) 'Synchronized routing of seasonal products through a production/distribution network', Central European Journal of Operations Research, Vol. 14, No. 2, pp.209-228. 
Brandao, J. (2009) 'A deterministic tabu search algorithm for the fleet size and mix vehicle routing problem', European Journal of Operational Research, Vol. 195, No. 3, pp.716-728.

Brandao, J. (2011) 'A tabu search algorithm for the heterogeneous fixed fleet vehicle routing problem', Computers \& Operations Research, Vol. 38, No. 1, pp.140-151.

Choi, E. and Tcha, D-W. (2007) 'A column generation approach to the heterogeneous fleet vehicle routing problem', Comput. Oper. Res., Vol. 34, No. 7, pp.2080-2095.

Clarke, G. and Wright, J. (1964) 'Scheduling of vehicles from central depot to number of delivery points', Operations Research, Vol. 12, No. 4, pp.568-581.

Drexl, M. (2012) 'Rich vehicle routing in theory and practice', Logistics Research, Vol. 5, Nos. 1-2, pp.47-63.

Euchi, J. and Chabchoub, H. (2010) 'A hybrid tabu search to solve the heterogeneous fixed fleet vehicle routing problem', Logistics Research, Vol. 2, No. 1, pp.3-11.

Fischetti, M., Toth, P. and Vigo, D. (1994) 'A branch-and-bound algorithm for the capacitated vehicle-routing problem on directed-graphs', Operations Research, Vol. 42, No. 5, pp.846-859.

Fisher, M. and Jaikumar, R. (1981) 'A generalized assignment heuristic for vehicle-routing', Networks, Vol. 11, No. 2, pp.109-124.

Gendreau, M., Laporte, G., Musaraganyi, C. and Taillard, E. (1999) 'A tabu search heuristic for the heterogeneous fleet vehicle routing problem', Computers \& Operations Research, Vol. 26, No. 12, pp.1153-1173.

Golden, B.L., Assad, A., Levy, L. and Gheysens, F. (1984) 'The fleet size and mix vehicle routing problem', Computers \& Operations Research, Vol. 11, No. 1, pp.49-66.

Golden, B.L., Assad, A.A. and Wasil, E.A. (2002) 'Routing vehicles in the real world: applications in the solid waste, beverage, food, dairy and newspaper industries', in Toth, P. and Vigo, D. (Eds.): The Vehicle Routing Problem, pp.245-286, SIAM Monographs on Discrete Mathematics and Applications, Philadelphia, USA.

Golden, B.L., Raghavan, S. and Wasil, E.A. (2008) The Vehicle Routing Problem: Latest Advances and New Challenges, Vol. 43 of Operations Research/Computer Science Interfaces Series, Springer, New York, USA.

Hartl, R., Hasle, G. and Janssens, G. (2006) 'Special issue on rich vehicle routing problems', Central European Journal of Operations Research, Vol. 14, No. 2, pp.103-104.

Imran, A., Salhi, S. and Wassan, N.A. (2009) 'A variable neighborhood-based heuristic for the heterogeneous fleet vehicle routing problem', European Journal of Operational Research, Vol. 197, No. 2, pp.509-518.

Irnich, S. (2008) 'A unified modeling and solution framework for vehicle routing and local search-based metaheuristics', INFORMS Journal on Computing, Vol. 20, No. 2, pp.270-287.

Juan, A., Faulin, J., Ruiz, R., Barrios, B. and Caballe, S. (2010) 'The SR-GCWS hybrid algorithm for solving the capacitated vehicle routing problem', Applied Soft Computing, Vol. 10, No. 1, pp.215-224.

Juan, A.A., Faulin, J., Jorba, J., Riera, D., Masip, D. and Barrios, B. (2011) 'On the use of Monte Carlo simulation, cache and splitting techniques to improve the Clarke and Wright savings heuristics', JORS, Vol. 62, No. 6, pp.1085-1097.

Laporte, G. (2009) 'Fifty years of vehicle routing', Transportation Science, Vol. 43, No. 4, pp.408-416.

Laporte, G. and Nobert, Y. (1987) 'Exact algorithms for the vehicle routing problem', Annals of Discrete Mathematics, Vol. 31, No. 1, pp.147-184.

Laporte, G., Mercure, H. and Nobert, Y. (1986) 'An exact algorithm for the asymmetrical capacitated vehicle-routing problem', Networks, Vol. 16, No. 1, pp.33-46.

Li, F., Golden, B. and Wasil, E. (2007) 'A record-to-record travel algorithm for solving the heterogeneous fleet vehicle routing problem', Computers and Operation Research, Vol. 34, No. 9, pp.2734-2742. 
Li, X., Tian, P. and Aneja, Y. (2010) 'An adaptive memory programming metaheuristic for the heterogeneous fixed fleet vehicle routing problem', Transportation Research Part E: Logistics and Transportation Review, Vol. 46, No. 6, pp.1111-1127.

Lima, C., Goldbarg, M. and Goldbarg, E. (2004) 'A memetic algorithm for the heterogeneous fleet vehicle routing problem', Electronic Notes in Discrete Mathematics, Vol. 18, No. 1, pp.171-176.

Liu, S., Huang, W. and Ma, H. (2009) 'An effective genetic algorithm for the fleet size and mix vehicle routing problems', Transportation Research Part E: Logistics and Transportation Review, Vol. 45, No. 3, pp.434-445.

Marmion, M-E., Humeau, J., Jourdan, L. and Dhaenens, C. (2010) 'Comparison of neighborhoods for the HFF-AVRP', 2010 IEEE/ACS International Conference on Computer Systems and Applications (AICCSA), pp.1-7.

Nagata, Y. (2007) 'Edge assembly crossover for the capacitated vehicle routing problem', in Cotta, C. and van Hemert, J.I. (Eds.): 7th European Conference on Evolutionary Computation in Combinatorial Optimization, EvoCOP, Lecture Notes in Computer Science, Valencia, Springer, pp.142-153.

Oppen, J., Løkketangen, A. and Desrosiers, J. (2010) 'Solving a rich vehicle routing and inventory problem using column generation', Computers \& Operations Research, Vol. 37, No. 7, pp.1308-1317.

Penna, P., Subramanian, A. and Ochi, L. (2013) 'An iterated local search heuristic for the heterogeneous fleet vehicle routing problem', Journal of Heuristics, Vol. 19, No. 2, pp.201-232.

Pessoa, A., de Aragão, M.P. and Uchoa, E. (2008) 'Robust branch-cut-and-price algorithms for vehicle routing problems', in Golden, B., Raghavan, S. and Wasil, E. (Eds.): The Vehicle Routing Problem: Latest Advances and New Challenges, Vol. 43 of Operations Research/Computer Science Interfaces, pp.297-325., Springer, USA.

Pisinger, D. and Røpke, S. (2007) 'A general heuristic for vehicle routing problems', Computers \& Operations Research, Vol. 34, No. 8, pp.2403-2435.

Prescott-Gagnon, E., Desaulniers, G. and Rousseau, L. (2012) 'Heuristics for an oil delivery vehicle routing problem', Flexible Services and Manufacturing Journal, pp.1-24, Springer US, ISSN: 1936-6582, DOI: 10.1007/s10696-012-9169-9.

Prins, C. (2002) 'Efficient heuristics for the heterogeneous fleet multitrip VRP with application to a large-scale real case', J. Math. Model. Algorithms, Vol. 1, No. 2, pp.135-150.

Prins, C. (2009) 'Two memetic algorithms for heterogeneous fleet vehicle routing problems', Eng. Appl. Artif. Intell., Vol. 22, No. 6, pp.916-928.

Prive, J., Renaud, J. and Boctor, F. (2006) 'Solving a vehicle-routing problem arising in soft-drink distribution', Journal of the Operational Research Society, Vol. 57, No. 9, pp.1045-1052.

Renaud, J. and Boctor, F.F. (2002) 'A sweep-based algorithm for the fleet size and mix vehicle routing problem', European Journal of Operational Research, Vol. 140, No. 3, pp.618-628.

Rieck, J. and Zimmermann, J. (2010) 'A new mixed integer linear model for a rich vehicle routing problem with docking constraints', Annals of Operations Research, Vol. 181, No. 1, pp.337-358.

Rodríguez, A. and Ruiz, R. (2012) 'A study on the effect of the asymmetry on real capacitated vehicle routing problems', Computers \& Operations Research, Vol. 39, No. 9, pp.2142-2151.

Ruiz, R., Maroto, C. and Alcaraz, J. (2004) 'A decision support system for a real vehicle routing problem', European Journal of Operational Research, Vol. 153, No. 3, pp.593-606.

Subramanian, A., Penna, P., Uchoa, E. and Ochi, L. (2012) 'A hybrid algorithm for the heterogeneous fleet vehicle routing problem', European Journal of Operational Research, Vol. 221, No. 2, pp.285-295.

Taillard, E.D. (1999) 'A heuristic column generation method for the heterogeneous fleet vehicle routing problem', RAIRO - Operations Research, Vol. 33, No. 1, pp.1-14. 
Tarantilis, C., Kiranoudis, C. and Vassiliadis, V. (2004) 'A threshold accepting metaheuristic for the heterogeneous fixed fleet vehicle routing problem', European Journal of Operational Research, Vol. 152, No. 1, pp.148-158.

Toth, P. and Vigo, D. (2001) 'An overview of vehicle routing problems', The Vehicle Routing Problem, pp.1-26, SIAM - Society for Industrial and Applied Mathematics, Philadelphia.

Toth, P. and Vigo, D. (2002) 'Models, relaxations and exact approaches for the capacitated vehicle routing problem', Discrete Applied Mathematics, Vol. 123, Nos. 1-3, pp.487-512.

Vallejo, M., Vargas, P. and Corne, D. (2012) 'A fast approximative approach for the vehicle routing problem', 12th UK Workshop on Computational Intelligence (UKCI), UK, pp.1-8.

Vigo, D. (1996) 'A heuristic algorithm for the asymmetric capacitated vehicle routing problem', European Journal of Operational Research, Vol. 89, No. 1, pp.108-126.

Wassan, N.A. and Osman, I.H. (2002) 'Tabu search variants for the mix fleet vehicle routing problem', The Journal of the Operational Research Society, Vol. 53, No. 7, pp.768-782.

Wen, M., Clausen, J. and Larsen, J. (2010) Rich Vehicle Routing Problems and Applications, PhD thesis, DTU Management. 